\title{
Une loi pour réglementer les analyses génétiques humaines à compter du 1er avril 2007
}

\author{
Le $1^{\mathrm{er}}$ avril 2007, le Conseil fédéral mettra en vigueur la loi sur l'analyse généti- \\ que humaine (LAGH) ainsi que deux ordonnances et nommera une commission \\ d'experts. En outre, une ordonnance correspondante émanant du Départe- \\ ment fédéral de l'intérieur (DFI) entrera en vigueur à la même date. La loi \\ et les ordonnances susmentionnées réglementent les aspects essentiels de la \\ réalisation d'analyses humaines en vue de protéger la dignité humaine, d'em- \\ pêcher les abus et d'assurer la qualité des examens.
}

Cristina Benedetti

Les analyses à des fins de recherche sont toutefois soumises à la réglementation dans le domaine de la recherche.

Correspondance:

Cristina Benedetti,

pharm. dipl. féd. MPH

Office fédéral de la santé publique Unité de direction santé publique Division Biomédecine CH-3003 Berne

cristina.benedetti@bag.admin.ch
Les analyses génétiques humaines viennent élargir le panel de solutions diagnostiques, préventives et thérapeutiques disponibles pour soigner les maladies incurables à ce jour. Elles permettent également de détecter une prédisposition à des maladies avant l'apparition des symptômes cliniques et de déceler des défaillances génétiques avant la naissance. Autant de raisons de déclencher un débat sur la dimension éthique, psychologique et sociale de cette problématique. La connaissance d'une prédisposition génétique à une maladie peut certes être mise au service de la prévention mais elle peut également soulever des questions délicates liées à la planification familiale et à la conception de la vie. Des tiers pourraient également en faire un usage abusif, p.ex. en refusant des prestations d'assurance à la personne concernée ou en compromettant ses opportunités professionnelles. La loi fédérale sur l'analyse génétique humaine (LAGH), qui entrera en vigueur le $1^{\text {er }}$ avril 2007 , réglemente:

- la réalisation des analyses génétiques humaines dans les domaines de la médecine, du travail, de l'assurance et de la responsabilité civile ainsi que

- l'établissement de profils d'ADN pour la détermination de la filiation ou l'identification de personnes, à l'exception de ceux utilisés dans des procédures pénales et pour l'identification de personnes inconnues ou disparues (la loi sur les profils d'ADN s'applique dans ces deux cas).
La LAGH vise à protéger la dignité humaine, à empêcher les abus et à assurer la qualité des analyses. Son champ d'application s'étend aux analyses cytogénétiques et moléculaires effectuées sur l'être humain dans le but de déterminer des caractéristiques du patrimoine génétique héréditaires ou acquises pendant la phase embryonnaire et à toutes les autres analyses de laboratoire qui visent à obtenir de manière directe ces mêmes informations. Pour ce qui est des analyses prénatales, celles visant à évaluer un risque englobent également les examens de l'embryon par des analyses ultrasonographiques. En revanche, les analyses des caractéristiques acquises au cours de la vie (mutations somatiques) et les analyses génétiques à des fins de recherche ne sont pas soumises aux dispositions de ladite loi. Ces analyses ne sont par conséquent pas soumises à autorisation*.

\section{Prescription de I'obligation d'informer}

En ce qui concerne les analyses génétiques, la LAGH fixe que nul ne doit être discriminé en raison de son patrimoine génétique (interdiction de discriminer). Les personnes concernées doivent en outre donner leur consentement pour l'analyse et disposent du droit à ne pas être informées. La loi accorde également une grande importance à la qualité des analyses, car ces dernières sont complexes, leurs résultats difficiles à interpréter, et leur réalisation peut être lourde de conséquences pour les personnes concernées et leurs pro- 
Membres de la Commission d'experts Sabina Gallati, présidente (Berne); Armand Bottani (Genève); Walter Bär (Zurich); Matthias Baumgartner (Zurich); Gieri Cathomas (Liestal); Bernice Elger (Genève); Susan Gerber (Berne); Andreas Huber (Aarau); Peter Miny (Bâle); Michael Morris (Genève); Judith Pók Lundquist (Zurich); Nicole ProbstHensch (Zurich).

ches. Ce niveau de qualité élevé doit non seulement être respecté lors de la réalisation des analyses par les laboratoires, mais également tout au long de la procédure d'information et de conseil de la personne concernée. La loi fixe ainsi les aspects sur lesquels la personne concernée doit être conseillée de manière non-directive par une personne qualifiée, lors d'une analyse génétique présymptomatique ou prénatale. La loi impose également aux cantons de veiller à ce que des services indépendants d'information et de conseil en matière d'analyse prénatale soient en place.

\section{La qualité garantie par la qualification}

Seuls les laboratoires autorisés par l'Office fédéral de la santé publique (OFSP) sont habilités à réaliser des analyses génétiques. Les éléments déterminants pour l'octroi d'une autorisation sont la qualification du chef de laboratoire, l'application d'un système de gestion de la qualité approprié et la réalisation d'un contrôle de qualité externe régulier. L'étendue des analyses génétiques que le laboratoire est habilité à réaliser dépend de la qualification professionnelle du chef de laboratoire. Ainsi, un laboratoire dont le chef dispose du titre «Spécialiste FAMH en analyses de génétique médicale» est habilité à exécuter toutes les analyses génétiques. En revanche, un laboratoire ne peut exécuter que certaines analyses génétiques, fixées par voie d'ordonnance, lorsque son responsable est titulaire du titre «Spécialiste FAMH en analyses de chimie clinique», «Spécialiste FAMH en analyses d'hématologie», «Spécialiste FAMH en analyses d'immunologie clinique», «Spécialiste FAMH en analyses de laboratoire médical (pluridisciplinaire)» ou «Spécialiste FMH en pathologie justifiant d'une formation approfondie en pathologie moléculaire». La liste des analyses est fixée par le Département fédéral de l'intérieur, en analogie avec la réglementation en vigueur dans le domaine de l'assurance obligatoire des soins. L'or- ganisation d'inspections annoncées ou inopinées permet de vérifier que les dispositions sont respectées. Dans les laboratoires accrédités, les contrôles du Service d'accréditation suisse se substituent aux inspections. Cette procédure permet d'éviter les doublons onéreux.

Les conditions et la procédure d'octroi de l'autorisation et les obligations des laboratoires sont fixées dans l'ordonnance sur l'analyse génétique humaine. Les laboratoires doivent déposer leur demande auprès de l'OFSP d'ici au 30 juin 2007.

\section{Dépistage}

La loi définit les examens de dépistage comme étant les analyses génétiques proposées de manière systématique à l'ensemble de la population ou à un groupe déterminé de personnes au sein de celle-ci. Le dépistage ne peut avoir lieu que si le programme a été autorisé par l'OFSP. La loi définit trois conditions nécessaires à l'octroi d'une telle autorisation. Il faut:

- qu'un traitement précoce ou des mesures prophylactiques existent;

- que la méthode d'analyse fournisse des résultats fiables et

- qu'un conseil génétique adéquat soit offert.

Les sociétés de médecine spécialisées, les autorités sanitaires cantonales et les hôpitaux universitaires figurent entre autres parmi les requérants de ce type d'autorisation. Les examens de dépistage déjà en cours au moment de l'entrée en vigueur de la loi ne sont pas soumis au régime d'autorisation.

\section{Etablissement de profils d'ADN}

La LAGH prescrit une procédure de reconnaissance des laboratoires qui établissent des profils d'ADN visant à déterminer la filiation ou l'identité d'une personne en dehors d'une procédure pénale. Les conditions et la procédure de reconnaissance sont réglementées dans l'ordonnance sur l'établissement de profils d'ADN en matière civile et administrative. Le Département fédéral de justice et police est compétent pour la reconnaissance et l'Office fédéral de la police (fedpol) est chargé de fournir des renseignements en la matière.

\section{Commission d'experts}

Lors de l'entrée en vigueur de la loi et des ordonnances y afférentes, le $1^{\text {er }}$ avril 2007, le Conseil fédéral instituera également une Commission d'experts pour les analyses génétiques humaines. La commission en question est chargée de suivre attentivement l'évolution scientifique et pra- 
tique en matière d'analyses génétiques. Il lui incombe aussi d'émettre des recommandations et de signaler suffisamment tôt les problèmes et les lacunes de la législation dans ce domaine. Elle comprend douze membres possédant des connaissances approfondies et de l'expérience en génétique humaine, en pédiatrie, en gynécologie, en médecine légale, en pathologie moléculaire et en analyses de génétique médicale.

\section{Pour plus d'informations}

Sur l'autorisation de réaliser des analyses génétiques et des examens de dépistage: Office fédéral de la santé publique, Unité de direction Santé publique, Division Biomédecine, 3003 Berne, tél. 0313235154 (secrétariat), fax 03132262 33, e-mail: genetictesting@bag.admin.ch, Internet: www.bag.admin.ch/genetictesting.
Sur la reconnaissance des laboratoires établissant des profils d'ADN: Office fédéral de la police, Division Services, Service de reconnaissance OACA, 3003 Berne, fax 03132414 10, www. fedpol.admin.ch/fedpol/fr/home/dienstleistun gen.html.

\section{Site d'informations}

L'OFSP et la fedpol créeront un site Internet consacré à l'exécution de la nouvelle législation qui sera mis en ligne le $1^{\mathrm{er}}$ avril 2007. Les requérants d'autorisation y trouveront des informations utiles et pourront télécharger les formulaires de demandes. 